\title{
Young women partition fatty acids towards ketone body production rather than VLDL-TAG synthesis, compared with young men
}

\author{
Kyriakoula Marinou ${ }^{1,2}$, Martin Adiels ${ }^{3}$, Leanne Hodson $^{1}$, Keith N. Frayn ${ }^{1}$, Fredrik Karpe ${ }^{1}$ \\ and Barbara A. Fielding ${ }^{1 *}$ \\ ${ }^{1}$ Oxford Centre for Diabetes, Endocrinology and Metabolism, University of Oxford, Churchill Hospital, Oxford OX3 7LJ, UK \\ ${ }^{2}$ Department of Experimental Physiology, Athens University School of Medicine, Athens, Greece \\ ${ }^{3}$ Wallenberg Laboratory, Sahlgrenska Center for Cardiovascular and Metabolic Research, Göteborg University, \\ Göteborg, Sweden
}

(Received 24 June 2010 - Revised 30 September 2010 - Accepted 5 October 2010 - First published online 21 January 2011)

\section{Abstract}

Before the menopause, women are relatively protected against CVD compared with men. The reasons for this sex difference are not completely understood, but hepatic fatty acid metabolism may play a role. The present study aimed to investigate the utilisation of plasma NEFA by the liver and to determine whether they are partitioned differently into ketone bodies and VLDL-TAG in healthy, lean young men and women. Volunteers were studied during a prolonged overnight fast $(12-19 \mathrm{~h})$ using an intravenous infusion of [U- ${ }^{13} \mathrm{C}$ ]palmitate. After $12 \mathrm{~h}$ fasting, the women had a more advantageous metabolic profile with lower plasma glucose $(P<0 \cdot 05)$ and TAG $(P<0.05)$ but higher plasma NEFA $(P<0.05)$ concentrations. Plasma 3-hydroxybutyrate $(3$-OHB) concentrations rose more in women than in men, and the transfer of ${ }^{13} \mathrm{C}$ from $\left[\mathrm{U}_{-}{ }^{13} \mathrm{C}\right]$ palmitate to plasma $\left[{ }^{13} \mathrm{C}\right] 3$-OHB reached a plateau $6-7 \mathrm{~h}$ after the start of the infusion in women but was still increasing at $6 \mathrm{~h}$ in men. This implies a slower 3-OHB production rate and/or dilution by other precursor pools in men. In women, the high isotopic enrichment of plasma 3-OHB suggested that systemic plasma fatty acids were the major source of 3 -OHB production. However, in men, this was not observed during the course of the study $(P<0 \cdot 01)$. There were no sex differences for the incorporation of ${ }^{13} \mathrm{C}$ into $\mathrm{VLDL}_{1^{-}}$or $\mathrm{VLDL}_{2}$-TAG. The ability of young women to partition fatty acids towards ketone body production rather than VLDL-TAG may contribute to their more advantageous metabolic profile compared with young men.

\section{Key words: 3-Hydroxybutyrate: Stable isotopes: Esterification}

Premenopausal women are relatively protected against CVD compared with men ${ }^{(1)}$, but the reasons for this sex difference are not completely understood. An understanding of the underlying mechanisms for this difference may help us to prevent worsening of cardiovascular risk factors in men and women in later life. There is evidence for some sex differences in the plasma metabolic profile in young adults. Short-term fasting reveals sex differences in people who are young and lean; after fasting, women have lower plasma glucose and higher plasma NEFA concentrations than men, as summarised in Soeters et $a l^{(2)}$. The ketone body 3-hydroxybutyrate (3-OHB) is an important energy source for the brain and other tissues during prolonged fasting. Under starving conditions, it is considered to act as a central signal and energy-providing substrate involved in the regulation of food intake ${ }^{(3)}$. Also, it seems that 3-OHB has an anti-lipolytic effect and is a ligand for the nicotinic acid receptor. In this way, its effect is similar to nicotinic acid ${ }^{(4)}$. However, to the best of our knowledge, sex comparisons of plasma 3-OHB concentrations have rarely been reported in healthy, lean young men and women. The difference in blood 3-OHB concentrations has been reported to be greater in response to fasting with significantly higher concentrations in women than in men after $30 \mathrm{~h}$ fasting, but plasma insulin concentrations were similar ${ }^{(5)}$. After an overnight fast, plasma TAG concentrations in women were lower than those in men, but this difference did not reach statistical significance and did not change during a further $10 \mathrm{~h}$ of fasting ${ }^{(6)}$. However, plasma VLDL-TAG concentrations were lower in women ${ }^{(6)}$ in response to the extended fasting. The results from that study suggested that the liver in women secretes fewer but TAG-richer VLDL particles than the liver in men, and that clearance was faster in women. There are two major classes of VLDL secreted by the liver: $\mathrm{VLDL}_{1}$ is larger and more TAG-rich than $\mathrm{VLDL}_{2}$.

Abbreviations: 3-OHB, 3-hydroxybutyrate; MPE, mole percent excess; $R_{\mathrm{a}}$, rate of appearance; TTR, tracer:tracee ratio.

*Corresponding author: Dr B. A. Fielding, email barbara.fielding@ocdem.ox.ac.uk 
The latter can either be secreted directly from the liver or be formed by the peripheral hydrolysis of $\mathrm{VLDL}_{1}$. These two classes of lipoproteins have different properties; in subjects with type 2 diabetes, the secretion of $\mathrm{VLDL}_{1}$ is associated with liver fat content, hypertriacylglycerolaemia and atherogenic risk $^{(7)}$. VLDL-TAG production represents the export of fatty acids partitioned towards esterification and secretion rather than storage or oxidation in the liver. Therefore, the balance between the pathways of esterification and oxidation in men and women may be important for the development of CVD risk factors.

The aim of the present study was to investigate sex differences in plasma metabolites and hepatic fatty acid metabolic partitioning in the liver in response to an extended overnight fast. This enabled us to stress metabolic pathways in order to investigate sex differences in young people before the onset of traditional cardiovascular risk factors. Since systemic NEFA are the major substrate for VLDL-TAG and 3-OHB production, we aimed to follow the transfer of a stable isotope label from plasma NEFA into the products of hepatic metabolism.

\section{Materials and methods}

\section{Subjects}

Healthy, lean young men and women ( $n$ 12) were studied, and matched for age and BMI with no significant difference in waist circumference, but the women had a greater percentage of body fat (Table 1). Subjects, recruited from the wider Oxford community via advertisement, were free from any disease, weight stable and not taking any lipid-lowering medication or medication that would alter lipid metabolism. The studies were performed during the first week (follicular phase) of the menstrual cycle and conducted according to the guidelines laid down in the Declaration of Helsinki. All procedures involving human subjects were approved by the Oxfordshire Clinical

Table 1. Volunteer characteristics and baseline overnight fasting (12 h) plasma metabolite concentrations

(Mean values with their standard errors, $n 6$ )

\begin{tabular}{|c|c|c|c|c|}
\hline & \multicolumn{2}{|c|}{ Men } & \multicolumn{2}{|c|}{ Women } \\
\hline & Mean & SE & Mean & SE \\
\hline Age (years) & $25 \cdot 5$ & 4.4 & $22 \cdot 5$ & $2 \cdot 2$ \\
\hline BMI $\left(\mathrm{kg} / \mathrm{m}^{2}\right)$ & 21.5 & 2.5 & $21 \cdot 0$ & 1.6 \\
\hline Waist $(\mathrm{cm})$ & $77 \cdot 3$ & $6 \cdot 7$ & $72 \cdot 8$ & $4 \cdot 1$ \\
\hline Fat mass ( $\%$ body wt) & $12 \cdot 6$ & $2 \cdot 1$ & $22 \cdot 5^{\star \star \star}$ & $3 \cdot 9$ \\
\hline Body wt $(\mathrm{kg})$ & $70 \cdot 65$ & $3 \cdot 7$ & $61 \cdot 63^{*}$ & 1.59 \\
\hline NEFA $(\mu \mathrm{mol} / \mathrm{l})$ & 296 & 26 & $557^{\star}$ & 88 \\
\hline 3-OHB ( $\mu \mathrm{mol} / \mathrm{l})$ & 39 & 7 & 64 & 12 \\
\hline TAG $(\mu \mathrm{mol} / \mathrm{l})$ & 1145 & 146 & $870^{\star}$ & 93 \\
\hline Glucose (mmol/l) & $5 \cdot 1$ & 0.8 & $4 \cdot 8^{*}$ & 0.13 \\
\hline Insulin (pmol/l) & $77 \cdot 8$ & $8 \cdot 33$ & $56 \cdot 95$ & $12 \cdot 5$ \\
\hline $\mathrm{VLDL}_{1}-{ }^{-T A G}(\mu \mathrm{mol} / \mathrm{l})$ & 388 & 113 & 255 & 65 \\
\hline $\mathrm{VLDL}_{2}-\mathrm{TAG}(\mu \mathrm{mol} / \mathrm{l})$ & 207 & 40 & $129^{*}$ & 15 \\
\hline
\end{tabular}

3-OHB, 3-hydroxybutyrate.

Mean values were significantly different from those of men: ${ }^{\star} P<0.05$, ${ }^{\star \star \star} P<0.001$.
Research Ethics Committee. Written informed consent was obtained from all subjects.

\section{Study protocol}

Before the study day, subjects were asked to avoid foodstuffs naturally enriched in ${ }^{13} \mathrm{C}$ for $48 \mathrm{~h}$ and refrain from strenuous exercise and alcohol for $24 \mathrm{~h}$. On the evening before the study, subjects were required to eat a low-fat meal. On the day of the study, subjects arrived at the clinical research unit after an overnight fast. Fat mass was measured by bioelectrical impedance analysis, and fat-free mass was calculated as the difference between total body weight and fat mass. A cannula was inserted into an antecubital vein, and a baseline blood sample was taken for background isotopic enrichment. At time 0, a continuous intravenous infusion $(0.04 \mu \mathrm{mol} / \mathrm{kg}$ per $\mathrm{min})$ of potassium $\left[\mathrm{U}^{13} \mathrm{C}\right]$ palmitate (isotope purity $98 \%$; Cambridge Isotope Laboratories, Inc., Andover, MA, USA) complexed to human albumin was started, to label the plasma NEFA pool. This was continued until the end of the experiment (at $7 \mathrm{~h}$ ).

\section{Analyses}

Whole blood was collected into heparinised syringes (Sarstedt, Leicester, UK), plasma was rapidly separated by centrifugation at $4^{\circ} \mathrm{C}$, and plasma NEFA, VLDL-TAG and 3-OHB concentrations were determined enzymatically, as described previously ${ }^{(8,9)}$. Plasma insulin concentrations were measured by RIA ${ }^{(9)}$.

In ten subjects, $\mathrm{VLDL}_{1}$ (Svedberg flotation rate $\left(S_{\mathrm{f}}\right)$ 60-400) and $\mathrm{VLDL}_{2}\left(S_{\mathrm{f}} 20-60\right)$ were isolated by sequential flotation using density gradient ultracentrifugation in an SW40Ti swinging bucket rotor (Beckman Instruments, Palo Alto, CA, USA) at $40000 \mathrm{rpm}$ at $15^{\circ} \mathrm{C}$ for $4 \mathrm{~h}$ for $S_{\mathrm{f}}$ 60-400 lipoproteins and for a further $16 \mathrm{~h}$ to float $S_{\mathrm{f}}$ 20-60 lipoproteins.

\section{Fatty acid analysis and isotopic enrichment}

Fatty acid methyl esters were prepared from NEFA, VLDL $1^{-}$ and $\mathrm{VLDL}_{2}$-TAG fractions and isotopic enrichment was measured by GC and GC-MS, respectively, as described previously $^{(9)}$. Specific fatty acid concentrations were determined by multiplying the proportion of the specific fatty acid by the corresponding plasma concentration as determined enzymatically for plasma NEFA, $\mathrm{VLDL}_{1^{-}}$and $\mathrm{VLDL}_{2}$-TAG.

Isotopic enrichment from $\left[\mathrm{U}^{13} \mathrm{C}\right.$ ]fatty acids appearing in 3-OHB in deproteinised plasma was measured using a modified method of Beylot et al. ${ }^{(10)}$. Solutions of $\left[2,4-{ }^{13} \mathrm{C}_{2}\right] 3-\mathrm{OHB}$ (Cambridge Isotope Laboratories, Inc.) and unenriched $3-\mathrm{OHB}$ were prepared in $4 \%$ perchloric acid and diluted in deionised water to make an enrichment standard curve $(100 \mu \mathrm{mol} / \mathrm{l})$. Plasma samples $(0.5 \mathrm{ml})$ were 
deproteinised with $1 \mathrm{ml}$ perchloric acid (70 g/1). Duplicate $1 \mathrm{ml}$ aliquots of the supernatant were neutralised with $500 \mu \mathrm{l}$ neutralising reagent $\left(0 \cdot 5 \mathrm{M}-\mathrm{KHCO}_{3}\right.$ and $\left.\mathrm{K}_{2} \mathrm{CO}_{3}\right)$ on ice. After centrifugation, $500 \mu \mathrm{l}$ aliquots of the deproteinised plasma were pipetted into $10 \mathrm{ml}$ glass tubes and acidified to $\mathrm{pH} 1$ with $1 \mathrm{M}-\mathrm{HCl}$. After prior addition of $50 \mu \mathrm{l}$ neutralising reagent, $500 \mu \mathrm{l} 3$-OHB standards were acidified in the same manner. The 3 -OHB from samples and standards was extracted into $4.5 \mathrm{ml}$ ethyl acetatediethyl ether $(1: 1, \mathrm{v} / \mathrm{v})$ in $10 \mathrm{ml}$ glass tubes by mixing by hand for $2 \mathrm{~min}$ and then by rotary mixing for $1 \mathrm{~h}$. The aqueous and organic phases were separated by centrifugation, and the upper organic phase was evaporated to dryness under $\mathrm{N}_{2}$ at room temperature. $t$-Butyl dimethylsilyl derivatives of 3-OHB were prepared by the addition of $20 \mu \mathrm{l}$ pyridine and $20 \mu \mathrm{l} N$-(tert-butyldimethylsilyl)- $N$-methyltrifluoroacetamide $+1 \% t$-butyl dimethylchlorosilane. After incubation for at least $45 \mathrm{~min}$ at room temperature, the contents of the tubes were transferred to GC vials and analysed by GC-MS. The GC was equipped with a $30 \mathrm{~m}$ capillary column with a $5 \%$ diphenyl$95 \%$ dimethyl polysiloxane stationary phase (inner diameter $0.32 \mathrm{~mm}$, film thickness $0.25 \mu \mathrm{m}$; Thames Restek, Saunderton, UK). The 5890 GC was coupled to a $5973 \mathrm{~N}$ MSD (Agilent Technologies, Stockport, UK). Ions with mass-to-charge ratios $(\mathrm{m} / \mathrm{z})$ of $275(\mathrm{M}+0), 277(\mathrm{M}+2)$ were determined by selected ion monitoring. We assume that the latter corresponds with the formation of $3-\mathrm{OHB}$ from one labelled $\left[{ }^{13} \mathrm{C}_{2}\right]$ acetyl-CoA molecule, derived from $\left[\mathrm{U}_{-}{ }^{13} \mathrm{C}\right]$ palmitic acid via $\beta$-oxidation. Initially, we selected ions of $m / z 279(\mathrm{M}+4)$ but the GC-MS was not sufficiently sensitive to consistently detect a peak.

The tracer:tracee ratio (TTR) of a baseline measurement (before the administration of the stable isotope tracer) was subtracted from each sample's TTR to account for natural abundance. The TTR for $\left[\mathrm{U}_{-}{ }^{13} \mathrm{C}\right]$ palmitate were multiplied by the corresponding palmitate concentration in plasma NEFA and $\mathrm{VLDL}_{1^{-}}$and $\mathrm{VLDL}_{2}$-TAG to give tracer concentrations. Likewise, the TTR for $\left[{ }^{13} \mathrm{C}\right] 3-\mathrm{OHB}$ $(M+2):(M+0)$ were multiplied by the corresponding plasma 3-OHB concentrations to give tracer concentrations. Mole percent excess (MPE) was calculated from the TTR using the formula MPE $=1 /(1+1 /$ TTR $)$.

\section{Calculations}

The proportion of systemic plasma NEFA contributing to VLDL-TAG at $7 \mathrm{~h}$ was calculated as described previously $^{(11)}$. The remainder are assumed to be fatty acids from splanchnic sources, including those from visceral fat lipolysis, liver fat and de novo lipogenesis.

Whole-body rate of appearance $\left(R_{\mathrm{a}}\right)$ of NEFA was calculated using the isotopic enrichment in the plasma after an intravenous infusion of the $\left[\mathrm{U}_{-}{ }^{13} \mathrm{C}\right]$ palmitate ${ }^{(12)}$ during the last $5 \mathrm{~h}$ of the infusion. The rate of disappearance of
NEFA was calculated as $R_{\mathrm{a}} \mathrm{NEFA}-\mathrm{d} Q / \mathrm{d} t$, where $\mathrm{d} Q$ is the change in the amount of tracee with time ${ }^{(12)}$

The classic mathematical modelling of 3-OHB production could not be performed because the level of enrichment in 3-OHB did not allow for calculation of the precursor pool enrichment. However, we wished to examine the extent to which the enrichment of $\left[{ }^{13} \mathrm{C}\right]$ in plasma 3-OHB reflected the synthesis from systemic NEFA. Therefore, we estimated the maximal enrichment (MPE) in 3 -OHB for $(M+2)$ at $7 \mathrm{~h}$, assuming that the precursor for 3-OHB production was only from the plasma NEFA pool. Since we infused a palmitic acid tracer, we assumed that the enrichment in the mitochondrial acetyl-CoA pool would be diluted by (non-selective) contributions from other specific fatty acids. Therefore, we accounted for the number of carbon atoms in each fatty acid species and their relative proportions in the NEFA pool for each participant. The plasma enrichment of $\left[\mathrm{U}^{13}{ }^{13} \mathrm{C}\right]$ palmitate multiplied by the dilution factor gave an estimation of the $\left[{ }^{13} \mathrm{C}\right]$ acetyl-CoA enrichment in the mitochondria. The estimated maximal MPE was calculated from the formula for the probability of picking exactly one labelled and one unlabelled element, $2 P(1-P)$, where $P$ is the probability of an element to be labelled.

The $\mathrm{M}+4$ isotopomer for 3 -OHB in women at plateau enrichment was estimated from the calculated precursor enrichment (MPE) and binomial expansion ${ }^{(13)}$.

In order to determine an approximation of the relative partitioning of systemic plasma NEFA into 3-OHB and VLDL-TAG, we calculated the ratio of $\left[{ }^{13} \mathrm{C}\right] 3-\mathrm{OHB}$ : $\left[{ }^{13} \mathrm{C}\right]$ VLDL-TAG in the plasma, where $\left[{ }^{13} \mathrm{C}\right]$ is expressed in $\mu \mathrm{mol} / 1$.

\section{Statistical analysis}

Data were analysed using SPSS for Windows version 16 (SPSS, Chertsey, UK). Statistical significance was set at $P<0.05$. All data are presented as means with their standard errors. Repeated-measures ANOVA, with time and group as factors, was used to investigate the change between men and women over time while fasting. Comparisons between groups after the extended overnight fast ( $7 \mathrm{~h}$ ) were made using a Mann-Whitney test.

\section{Terminology}

Conventional terminology for stable isotope tracer techniques is used in the present paper ${ }^{(12)}$. The passage of a molecule through the GC-MS results in the production of a major ion of interest with mass M. An isotopomer is a molecule that has the same chemical composition but a different mass because it has an isotopic tracer incorporated somewhere in the molecule. Thus, the $\mathrm{M}$ isotopomer for 3 -OHB refers to an ion that has no ${ }^{13} \mathrm{C}$ tracer incorporated, and $M+2$ represents an ion that has a mass that is 2 atomic mass units greater than the $\mathrm{M}$ isotopomer; 
this is assumed to be due to the substitution of two ${ }^{12} \mathrm{C}$ atoms by two ${ }^{13} \mathrm{C}$ atoms.

\section{Results}

Differences in plasma variables between men and women after an overnight fast are given in Table 1 . After $12 \mathrm{~h}$ fasting, the men had a less advantageous metabolic profile in terms of higher plasma glucose $(P<0.05)$ and TAG $(P<0.05)$ concentrations but lower plasma NEFA $(P<0.05)$ concentrations. In order to verify that the twelve subjects whom we chose were representative of the wider community in this respect, we retrieved data from 266 men and 405 women (aged 30-50 years) from the Oxford Biobank ${ }^{(14)}$. All individuals were selected to have a BMI of less than $25 \mathrm{~kg} / \mathrm{m}^{2}$. In exact accordance with the six men and six women in the present study, the larger group of women had higher plasma NEFA concentrations (553 (SE 13) $v$ 475 (se 16) $\mu \mathrm{mol} / \mathrm{l} ; P<0.05$ ); the same plasma 3-OHB concentrations, lower plasma TAG concentrations (870 (SE 20) v. 1090 ( $\mathrm{SE} 40) \mu \mathrm{mol} / \mathrm{l} ; \quad P<0.05$ ); lower plasma glucose concentrations $(4.8$ (SE 0.0$) v .5 \cdot 2($ SE 0.0$) \mathrm{mmol} / \mathrm{l} ; P<0.05)$ and similar plasma insulin concentrations. After extension of the postabsorptive period during the $7 \mathrm{~h}$ of the present experiment, expected changes in plasma variables occurred (Fig. 1). Significant differences between men and women for glucose, TAG and NEFA were maintained, and the marginally higher values for $3-\mathrm{OHB}$ found in women after $12 \mathrm{~h}$ fasting $(P=0.078)$ became significant $(P=0.035)$. Plasma insulin concentrations decreased significantly with time $(P=0.02)$ but remained similar between men and women after prolonged fasting.

Concentrations of $\mathrm{VLDL}_{2}$-TAG but not $\mathrm{VLDL}_{1}$-TAG were significantly higher in men than in women (Table 1). We observed that $\mathrm{VLDL}_{1}$-TAG concentrations were higher than $\mathrm{VLDL}_{2}$-TAG in both sexes combined $(P=0.028)$. During prolonged fasting, the concentrations of $\mathrm{VLDL}_{1}{ }^{-}$ TAG decreased significantly (Fig. 1), but no further sex differences were revealed. There was a small decrease in $\mathrm{VLDL}_{2}$-TAG concentrations during the study, but this was not significant (females $P=0 \cdot 275$, males $P=0.663$ ).

The TTR of $\left[\mathrm{U}_{-}{ }^{13} \mathrm{C}\right]$ palmitate from the intravenous infusion reached a plateau in the plasma NEFA pool by $1 \mathrm{~h}$ in men and women (Fig. 2). $R_{\mathrm{a}} \mathrm{NEFA}$ ( $\mu \mathrm{mol} / \mathrm{min}$ ) was similar in men and women but lower in women when expressed as per $\mathrm{kg}$ fat mass (Table 2). When expressed according to fat-free mass, there was no difference between men and women (Table 2).

The fatty acid tracer was rapidly incorporated into plasma 3-OHB and VLDL-TAG. Whereas enrichment reached a plateau in plasma $3-\mathrm{OHB}$ at $6-7 \mathrm{~h}$ after the start of the infusion in women, it was still increasing at $6 \mathrm{~h}$ in men (Fig. 2). Plasma concentrations of $\left[{ }^{13} \mathrm{C}\right] 3-\mathrm{OHB}$ were correspondingly higher in women than in men during the course of the experiment $(P=0.007$ for the effect of sex, $P=0.004$ for the time $\times$ sex interaction).
In women, the observed isotopic enrichment of the fatty acid tracer in 3-OHB was close to that predicted, if systemic plasma fatty acids were the sole source of 3-OHB production (Table 2). In fact, the observed MPE reached $78 \%$ of the estimated maximal MPE, and thus only $22 \%$ of the 3-OHB could not be accounted for by the systemic NEFA sources. These values are in close agreement with the levels reached for $\mathrm{VLDL}_{1}$ - and $\mathrm{VLDL}_{2}$-TAG (Table 3) in these lean women. However, in men, the observed enrichment was less than half expected, implying either an alternative source of fatty acids or a slower rate of production. The incorporation of the $\left[{ }^{13} \mathrm{C}\right]$ tracer into $\mathrm{VLDL}_{1^{-}}$and $\mathrm{VLDL}_{2}$-TAG was not significantly different between men and women, in terms of either the TTR (Fig. 2) or the $\left[{ }^{13} \mathrm{C}\right] \mathrm{VLDL}-\mathrm{TAG}$ concentration (data not shown).

The ratio of $\left[{ }^{13} \mathrm{C}\right] 3-\mathrm{OHB}:\left[{ }^{13} \mathrm{C}\right] \mathrm{VLDL}-\mathrm{TAG}$ in the plasma was significantly higher in women than in men $(2 \cdot 73$ (SE 1.7) v. $0.66(\operatorname{se~} 0.30) ; P=0.027$ for $\mathrm{VLDL}_{1}$ and 3.24 (SE 1.6) v. $0.97(\operatorname{se~} 0.43) ; P=0.014$ for $\left.\mathrm{VLDL}_{2}\right)$.

As we have reported previously in the postprandial period for healthy people for total VLDL-TAG ${ }^{(11)}$, fatty acids from systemic plasma NEFA contributed a greater proportion of fatty acids to $\mathrm{VLDL}_{1}$-TAG and $\mathrm{VLDL}_{2}$-TAG than those from splanchnic sources, in men and women (Table 3). However, the proportion of systemic fatty acids was lower in men than in women for $\mathrm{VLDL}_{2}$-TAG, with a corresponding greater proportion from splanchnic sources.

\section{Discussion}

In the present study, we found significant differences in hepatic metabolism in healthy, lean young men and women. Sex comparisons of plasma 3-OHB in very young men and women have rarely been reported, but we are in agreement with previous findings of higher concentrations in young women after a $30 \mathrm{~h}$ fast ${ }^{(5)}$, and we found that a shorter period of fasting $(12-19 \mathrm{~h})$ revealed higher concentrations in women. We found no difference in plasma 3-OHB concentrations between men and women without the provocation of a prolonged fast, in either a young age group ( $n$ 12) or middle-aged individuals ( $n$ 671). Since ketone bodies are only produced in the liver, systemic concentrations, usually of $3-\mathrm{OHB}$, can be taken to reflect hepatic ketone body production. Lower 3-OHB concentrations have been reported in subjects with hyperlipidaemia ${ }^{(15,16)}$, obesity ${ }^{(17)}$ and insulin resistance $^{(11)}$. Therefore, lower 3-OHB concentrations seem to be associated with a less advantageous metabolic profile. These differences could be due to differences in production or clearance. The present findings suggest that in young lean women, a higher plasma concentration of 3-OHB, compared with men, is due to a greater production, although we did not measure production directly.

There were sex differences in the incorporation of carbon units from $\left[\mathrm{U}_{-}{ }^{13} \mathrm{C}\right]$ palmitate, representing systemic 

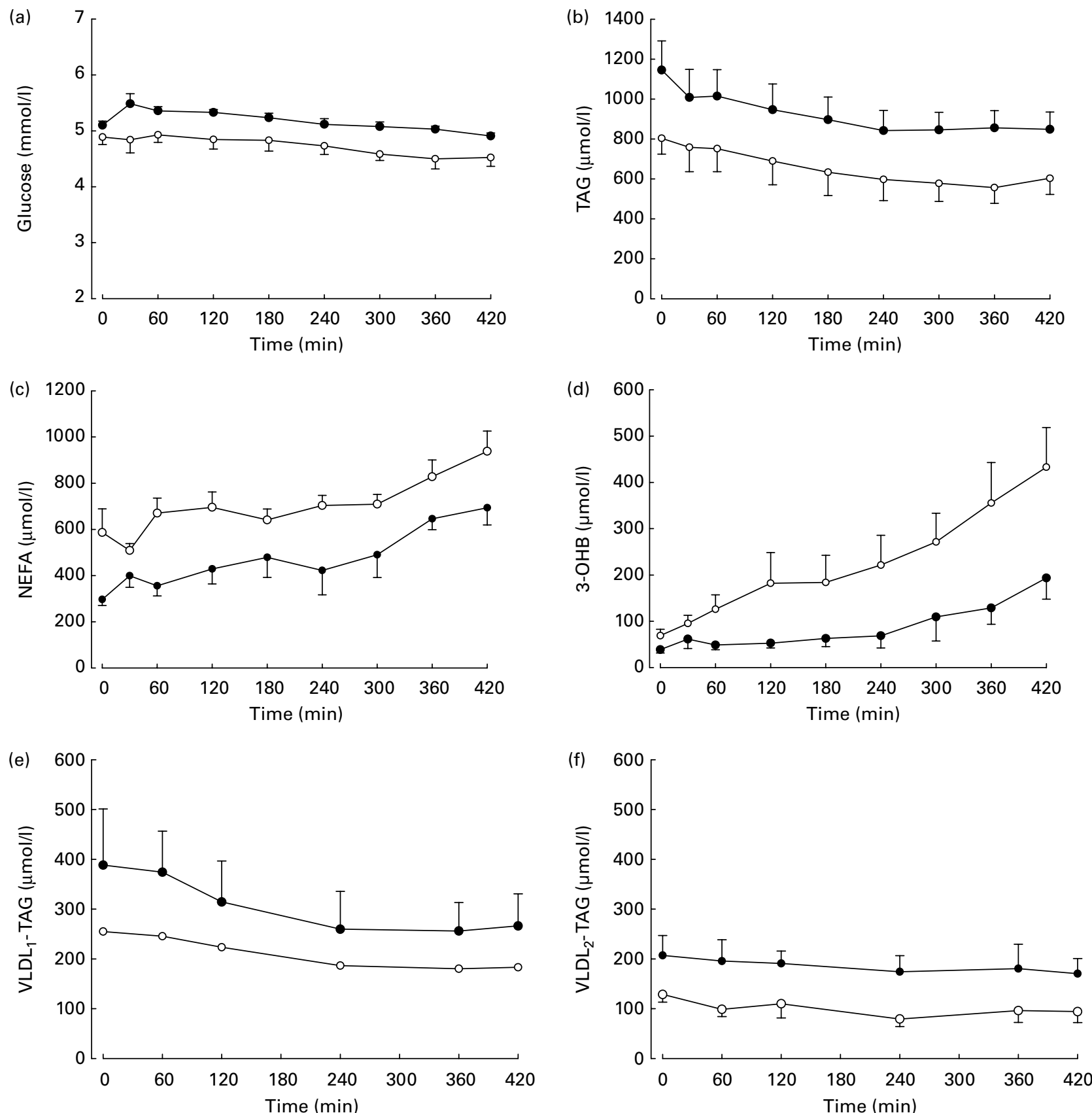

Fig. 1. Plasma concentrations of metabolites in response to continued overnight fasting and analysed by repeated-measures ANOVA. $\bullet$, Men ( $n$ 6); $\bigcirc$, women (n 6). Values are means, with standard errors represented by vertical bars. (a) Glucose, there was a significant effect of time $(P<0.05)$ and sex $(P<0.05)$. (b) TAG, there was a significant effect of time $(P<0.001)$ and a tendency for an effect of sex $(P=0.087)$. (c) NEFA, there were significant effects of time $(P<0.01)$ and sex $(P<0.01)$. (d) 3-Hydroxybutyrate (3-OHB), there were significant effects of time $(P<0.01)$ and sex $(P<0.05)$. (e) Plasma VLDL ${ }_{1}$ concentrations (men $(n 5)$, women $(n 5))$, in response to continued overnight fasting. There was a significant effect of time $(P<0.05)$. (f) $P$ lasma VLDL $L_{2}$ concentrations, there was a tendency for an effect of sex $(P=0.066)$.

fatty acids, into 3-OHB. The higher 3-OHB TTR and attainment of plateau enrichment in women suggests that new 3-OHB substantially contributes to total 3 -OHB. Thus, it could be hypothesised that women rapidly switch on 3-OHB production from systemic plasma NEFA, allowing plasma concentrations of 3 -OHB to increase rapidly and the TTR to flatten out. Men have a slower 3-OHB switch, and at $7 \mathrm{~h}$, the contribution of plasma NEFA is still increasing as a source of 3-OHB. However, it should be noted that the present findings are not necessarily representative of $n$-3 PUFA, which may partition away from $\beta$-oxidation in women ${ }^{(18)}$.

The reason for a greater ability of women to turn on 3 -OHB production is not clear. The production of ketone bodies is dependent, to a large extent, on the supply of plasma NEFA ${ }^{(19,20)}$. Plasma NEFA concentrations that increased experimentally lead to increased plasma concentrations of $3-\mathrm{OHB}^{(19,20)}$. Beylot et al. ${ }^{(21)}$ found that the 

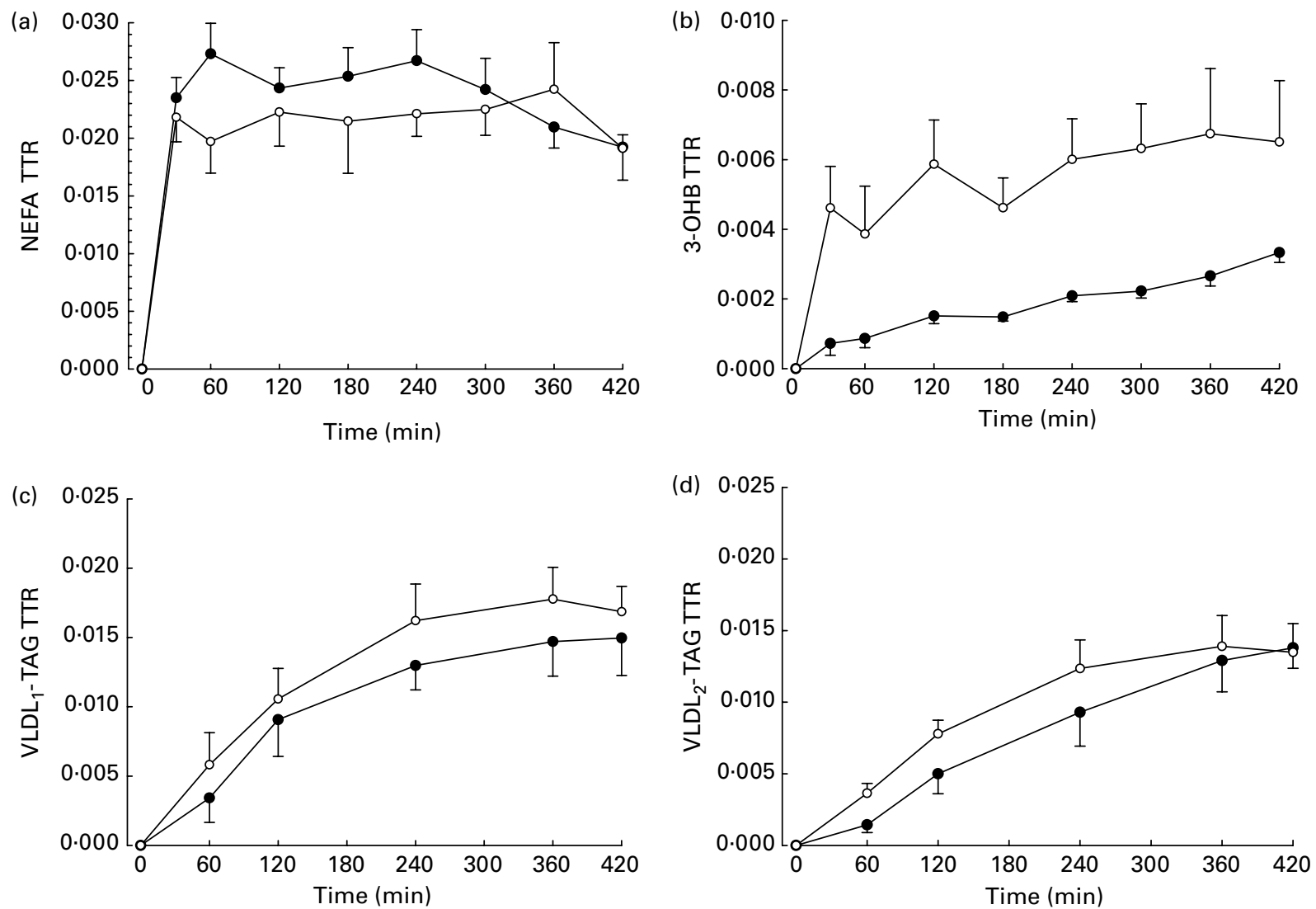

Fig. 2. Plasma tracer:tracee ratio (TTR) resulting from an intravenous infusion of $\left[\mathrm{U}-{ }^{13} \mathrm{C}\right]$ palmitate, starting at time 0 . $\bullet$, Men $(n$ 6); O, women $(n$ 6). (a) NEFA $(n 6)$, there were significant effects of time $(P<0.05)$, time $\times$ sex interaction $(P<0.05)$ and a tendency for an effect of sex $(P=0.086)$. (b) 3-Hydroxybutyrate (3-OHB, $n$ 5), there was a significant effect of time $(P<0.001)$ and sex $(P<0.05)$. (c) VLDL ${ }_{1}$, there was a significant effect of time $(P<0.001)$. (d) $V L D L_{2}$, there was a significant effect of time $(P<0.001)$. Values are means, with standard errors represented by vertical bars.

percentage conversion of plasma NEFA to 3-OHB increased in a linear fashion as the precursor concentration increased. This is thought to be due to reduced malonylCoA and reduced inhibition of carnitine palmitoyl transferase I, and could possibly account for the sex differences that we observed. A greater percentage conversion of plasma NEFA to 3-OHB may have occurred in the women of the present study, whose mean plasma NEFA concentration was almost twice that of men. Glucagon is also an important stimulator of ketone body production, but it is not clear if there is a sex difference. Glucagon levels have been reported as similar in men and women ${ }^{(5)}$, but in an older study by Merimee \& Fineberg ${ }^{(22)}$, plasma glucagon concentrations were significantly higher in premenopausal women than in men after a prolonged fast $(72 \mathrm{~h})$. Insulin reduces $3-\mathrm{OHB}$ production indirectly, via a direct effect on adipose tissue lipolysis, affecting the supply of NEFA. A direct effect on ketone body production has been described in some ${ }^{(23)}$ but not in all studies $^{(21,24)}$

Table 2. NEFA kinetics and 3-hydroxybutyrate $(3-\mathrm{OHB}){ }^{13} \mathrm{C}$ enrichment arising from the systemic NEFA pool at $420 \mathrm{~min}$ (Mean values with their standard errors, $n 6$ )

\begin{tabular}{lccccc}
\hline & \multicolumn{2}{c}{ Men } & & \multicolumn{2}{c}{ Women } \\
\cline { 2 - 4 } \cline { 3 - 5 } & Mean & SE & & Mean & SE \\
\hline$R_{\mathrm{a}}$ NEFA $(\mu \mathrm{mol} / \mathrm{min})$ & 594 & 101 & 533 & 61.5 \\
$R_{\mathrm{a}}$ NEFA $(\mu \mathrm{mol} / \mathrm{min}$ per kg fat mass) & 68.7 & 12.5 & & 38.1 & 2.59 \\
$R_{\mathrm{d}}$ NEFA $(\mu \mathrm{mol} / \mathrm{min}$ per kg fat-free mass) & 9.4 & 1.2 & & 11.2 & 0.2 \\
3-OHB estimated maximal MPE & 0.0082 & 0.0050 & & $0.0075 \S$ & 0.0010 \\
3-OHB observed MPE & $0.0033 \dagger$ & 0.00028 & & $0.0062 \S$ & 0.0017 \\
3-OHB observed/maximal MPE $(\%)$ & $42 \ddagger$ & 5.3 & & 78 & $10 \S$ \\
\hline
\end{tabular}

$R_{\mathrm{a}}$, rate of appearance; $R_{\mathrm{d}}$, rate of disappearance; MPE, mole percent excess. There was a significant time-by-sex effect: ${ }^{\star \star} P<0.005$.

Mean values were significantly different from those of women: $\dagger P<0.05, \ddagger P<0.01$. Effect of sex over a period of extended fasting (14-20 $\mathrm{h}$ ). $\S n 5$. 
Table 3. Contribution of splanchnic and systemic fatty acids to VLDL-TAG at $7 \mathrm{~h}$ in men and women (Mean values with their standard errors)

\begin{tabular}{|c|c|c|c|c|c|c|c|c|}
\hline & \multicolumn{4}{|c|}{$\operatorname{VLDL}_{1}$} & \multicolumn{4}{|c|}{$\mathrm{VLDL}_{2}$} \\
\hline & \multicolumn{2}{|c|}{ Splanchnic } & \multicolumn{2}{|c|}{ Systemic } & \multicolumn{2}{|c|}{ Splanchnic } & \multicolumn{2}{|c|}{ Systemic } \\
\hline & Mean & SE & Mean & SE & Mean & SE & Mean & SE \\
\hline \multicolumn{9}{|c|}{ Absolute contribution of different fatty acid sources to VLDL-TAG $(\mu \mathrm{mol} / \mathrm{l})$} \\
\hline Men & 61.5 & 22 & $205 \dagger$ & 53 & $52 \cdot 3$ & 12 & $118 \dagger$ & 20 \\
\hline Women & $27 \cdot 6$ & 22 & $155 \dagger$ & 56 & $12 \cdot 7$ & 7.4 & $81 \cdot 3 \dagger$ & 22 \\
\hline \multicolumn{9}{|c|}{ Relative contribution of different fatty acid sources to VLDL-TAG (\%) } \\
\hline Men & $25 \cdot 0$ & $6 \cdot 4$ & $75.0 \dagger$ & 6.4 & $30 \cdot 0$ & $2 \cdot 7$ & $70 \cdot 0 \dagger$ & $2 \cdot 7$ \\
\hline Women & $14 \cdot 7$ & $5 \cdot 5$ & $85.3+$ & $5 \cdot 5$ & 14.5 & $5 \cdot 5$ & $85 \cdot 5^{\star}$ & $5 \cdot 5$ \\
\hline
\end{tabular}

Although plasma NEFA concentrations were higher in women, they had a similar delivery of fatty acids into the systemic circulation than in men as determined by $R_{\mathrm{a}}$ NEFA. However, even though the women were lean, they had a significantly higher fat mass than the men, and when $R_{\mathrm{a}}$ NEFA was expressed in relation to fat mass, the $R_{\mathrm{a}}$ NEFA was significantly lower. This is in agreement with the recent publication of Mittendorfer et al. ${ }^{(25)}$ who reported a down-regulation of $R_{\mathrm{a}}$ NEFA per unit of fat mass in obesity. So, even within lean individuals, we were able to observe an effect of fat mass on $R_{\mathrm{a}}$ NEFA. In contrast to the present findings (no difference between men and women), the study of Mittendorfer et al. found a higher $R_{\mathrm{a}}$ NEFA ( $\left.\mu \mathrm{mol} / \mathrm{min}\right)$ in women, and reported similar plasma NEFA concentrations. A possible explanation for the observed difference between the two studies may be because we enrolled leaner, younger volunteers.

After $12 \mathrm{~h}$ fasting, the men in the present study had a more disadvantageous metabolic profile in terms of higher plasma glucose and TAG concentrations but lower plasma NEFA concentrations. Since the latter are generally thought of as an adverse CVD risk factor, especially in terms of obesity and diabetes ${ }^{(26)}$, this was unexpected. However, we have shown that a metabolic disadvantage of a low plasma NEFA concentration is the reduced ability of the liver to switch to ketone body production during short-term fasting, and in the long term, this could possibly lead to the development of fatty liver. Plasma NEFA is also a substrate for VLDL-TAG synthesis. In the present study, more than three quarters of VLDL-TAG were delivered from systemic plasma NEFA, in men and women. However, particularly in $\mathrm{VLDL}_{2}$, the contribution of systemic plasma NEFA to VLDL-TAG was lower in men than in women. This may have been due to a lower flux from systemic fatty acids and/or a greater contribution from splanchnic sources. The latter would include de novo lipogenesis, fatty acid from the lipolysis of visceral fat or from cytosolic storage pools.

We calculated an 'estimated' maximum ${ }^{13} \mathrm{C}$ enrichment in 3-OHB that would be achieved if systemic fatty acids were the sole precursor pool for 3-OHB production. This was similar for men and women. For women, the achieved value was $78 \%$ of the value expected, implying that systemic fatty acids were the major precursor pool for 3-OHB production. This is in line with the hypothesis that cytosolic TAG fatty acids may not provide substrate for ketone body production, suggesting compartmentalisation of the precursor pool of ketone bodies ${ }^{(27)}$. It also suggests that fatty acids from visceral fat did not substantially contribute to ketone body production in these lean young women. However, in men, the enrichment of ${ }^{13} \mathrm{C}$ in 3 -OHB was less than half the value expected. Consistent with this would be dilution of the precursor pool by non-systemic fatty acids, such as those from visceral fat. Alternatively, a lower 3-OHB production rate in men would mean that the maximum isotopic enrichment had not been reached during the course of the experiment. Parallel to these findings for 3-OHB isotopic enrichment, the VLDL isotopic enrichment in women was close to that of the plasma NEFA pool; thus over $85 \%$ of VLDL were derived from this pool. This suggests that in women, the precursor fatty acids for both metabolic pathways over the duration of the present study were approximately $80 \%$ from plasma NEFA, and implies that plasma NEFA were an immediate and major source of fatty acids for hepatic fatty acid metabolism, contributing equally to 3-OHB and VLDLTAG synthesis during a prolonged overnight fast. However, since there were no sex differences in VLDL isotopic enrichment, the implication is that women rapidly partition a greater proportion of systemic fatty acids into ketone bodies than men. In men, it cannot be ruled out that the same sources are used for 3-OHB and VLDL-TAG, but since the 3 -OHB enrichment is still rising at the end of the experiment, and the total 3-OHB pool is much lower than for women, it is clear either that the time course is considerably slower or that other sources of fatty acids are involved.

Ketone bodies may be infused intravenously to give kinetic information on ketone body production ${ }^{(21,23,28)}$, but the use of a fatty acid stable isotope tracer has not been utilised previously to investigate ketone body metabolism in humans. The disadvantage of the latter approach is that we were not able to do classic kinetic modelling, because the lack of detectable $M+4$ tracer 
labelling in 3-OHB did not allow for calculation of the precursor pool enrichment. Quantitatively, the $\mathrm{M}+4$ isotopomer would not contribute significantly to the total appearance of the palmitate tracer in 3-OHB. We calculated that in women, the enrichment (MPE) of the 3-OHB M +4 isotopomer was less than $0.5 \%$ of the $\mathrm{M}+2$ isotopomer. However, the results were informative. In particular, we were able to show that in women, systemic plasma NEFA was the main source of fatty acids for 3-OHB production, and that hepatic production of 3-OHB followed different kinetics in men and women. We calculated the $\left[{ }^{13} \mathrm{C}\right] 3-O H B:\left[{ }^{13} \mathrm{C}\right]$ VLDL-TAG ratio in the plasma, and found that mean values were greater than 2.5 in women. This illustrates the quantitative importance of ketone body production for NEFA turnover in women, in the early fasting period. Beylot et al. ${ }^{(21)}$ calculated that for a plasma 3-OHB concentration of $464 \mu \mathrm{mol} / 1,13 \%$ of plasma NEFA are converted to $3-\mathrm{OHB}$ in healthy young men. The present results would suggest an even higher rate of conversion in women.

The hepatic partitioning of fatty acids into ketone bodies or towards esterification is difficult to study in humans but may be an important metabolic regulatory point that warrants further study. We have shown that in young women, plasma fatty acids tend to be readily converted to ketone bodies after an extended overnight fast, possibly partly because of a higher systemic pool than in men. Moreover, there is evidence of preferential partitioning to ketone bodies rather than VLDL-TAG, at least in early starvation. From an evolutionary point of view, women may therefore have been better adapted to cope with periods without food. In modern times, the greater ability of women to oxidise plasma NEFA into ketone bodies may partly explain the fact that premenopausal women have a better metabolic profile than men and may help protect women against the accumulation of liver fat. Fatty acid partitioning could potentially be manipulated by lifestyle changes or pharmacological intervention.

\section{Acknowledgements}

This work was funded in part by the project 'Hepatic and adipose tissue and functions in the metabolic syndrome' (http://www.hepadip.org/), which is supported by the European Commission as an Integrated Project under the Sixth Framework Programme (Contract LSHM-CT-2005018734). Funding is also gratefully acknowledged from the European Atherosclerosis Society, Novo Nordisk and the Sahlgrenska Center for Cardiovascular and Metabolic Research funded by the Foundation for Strategic Research. We thank Jane Cheeseman, Louise Dennis and Siobhán McQuaid for expert help during the studies. We confirm no conflict of interest for the work reported here. K. M. carried out the studies, and performed laboratory and data analyses. M. A. analysed the data and helped with the manuscript preparation. L. H. performed laboratory analysis, data analysis and helped with the manuscript preparation. B. A. F. developed stable isotope methods, supervised laboratory work and took the lead on the manuscript preparation. F. K. supervised the studies and helped with the manuscript preparation. K. N. F., L. H., B. A. F. and F. K. designed the study.

\section{References}

1. Booth GL, Kapral MK, Fung K, et al. (2006) Relation between age and cardiovascular disease in men and women with diabetes compared with non-diabetic people: a populationbased retrospective cohort study. Lancet 368, 29-36.

2. Soeters MR, Sauerwein HP, Groener JE, et al. (2007) Genderrelated differences in the metabolic response to fasting. J Clin Endocrinol Metab 92, 3646-3652.

3. Laeger T, Metges CC \& Kuhla B (2010) Role of beta-hydroxybutyric acid in the central regulation of energy balance. Appetite 54, 450-455.

4. Taggart AK, Kero J, Gan X, et al. (2005) (D)-beta-Hydroxybutyrate inhibits adipocyte lipolysis via the nicotinic acid receptor PUMA-G. J Biol Chem 280, 26649-26652.

5. Haymond MW, Karl IE, Clarke WL, et al. (1982) Differences in circulating gluconeogenic substrates during short-term fasting in men, women, and children. Metabolism 31, 33-42.

6. Magkos F, Patterson BW, Mohammed BS, et al. (2007) Women produce fewer but triglyceride-richer very lowdensity lipoproteins than men. J Clin Endocrinol Metab 92, 1311-1318.

7. Adiels M, Taskinen MR, Packard C, et al. (2006) Overproduction of large VLDL particles is driven by increased liver fat content in man. Diabetologia 49, 755-765.

8. Humphreys SM \& Frayn KN (1988) Micro-method for preparing perchloric extracts of blood. Clin Chem 34, 1657.

9. Bickerton AS, Roberts R, Fielding BA, et al. (2007) Preferential uptake of dietary fatty acids in adipose tissue and muscle in the postprandial period. Diabetes 56, 168-176.

10. Beylot M, Beaufrere B, Normand S, et al. (1986) Determination of human ketone body kinetics using stable-isotope labelled tracers. Diabetologia 29, 90-96.

11. Hodson L, Bickerton AS, McQuaid SE, et al. (2007) The contribution of splanchnic fat to VLDL-triglyceride is greater in insulin resistant than insulin sensitive men and women: studies in the postprandial state. Diabetes 56, 2433-2441.

12. Wolfe RR \& Chinkes D (2005) Isotope Tracers in Metabolic Research. Hoboken: John Wiley, Sons, Inc.

13. Hellerstein MK, Christiansen M, Kaempfer S, et al. (1991) Measurement of de novo hepatic lipogenesis in humans using stable isotopes. J Clin Invest 87, 1841-1852.

14. Tan GD, Neville MJ, Liverani E, et al. (2006) The in vivo effects of the Pro12Ala PPARgamma2 polymorphism on adipose tissue NEFA metabolism: the first use of the Oxford Biobank. Diabetologia 49, 158-168.

15. Havel RJ, Kane JP, Balasse EO, et al. (1970) Splanchnic metabolism of free fatty acids and production of triglycerides of very low density lipoproteins in normotriglyceridemic and hypertriglyceridemic humans. J Clin Invest 49, 2017-2035.

16. Evans K, Burdge GC, Wootton SA, et al. (2008) Tissuespecific stable isotope measurements of postprandial lipid metabolism in familial combined hyperlipidaemia. Atherosclerosis 197, 164-170.

17. Vice E, Privette JD, Hickner RC, et al. (2005) Ketone body metabolism in lean and obese women. Metabolism $\mathbf{5 4}$, $1542-1545$. 
18. Burdge GC \& Wootton SA (2003) Conversion of alpha-linolenic acid to palmitic, palmitoleic, stearic and oleic acids in men and women. Prostaglandins Leukot Essent Fatty Acids 69, 283-290.

19. Grey NJ, Karl I \& Kipnis DM (1975) Physiologic mechanisms in the development of starvation ketosis in man. Diabetes $\mathbf{2 4}$, $10-16$.

20. Johnston DG \& Alberti KG (1982) Hormonal control of ketone body metabolism in the normal and diabetic state. Clin Endocrinol Metab 11, 329-361.

21. Beylot M, Picard S, Chambrier C, et al. (1991) Effect of physiological concentrations of insulin and glucagon on the relationship between nonesterified fatty acids availability and ketone body production in humans. Metabolism $\mathbf{4 0}$, $1138-1146$

22. Merimee TJ \& Fineberg SE (1973) Homeostasis during fasting. II. Hormone substrate differences between men and women. J Clin Endocrinol Metab 37, 698-702.
23. Keller U, Gerber PP \& Stauffacher W (1988) Fatty acidindependent inhibition of hepatic ketone body production by insulin in humans. Am J Physiol 254, E694-E699.

24. Miles JM, Haymond MW, Nissen SL, et al. (1983) Effects of free fatty acid availability, glucagon excess, and insulin deficiency on ketone body production in postabsorptive man. J Clin Invest 71, 1554-1561.

25. Mittendorfer B, Magkos F, Fabbrini E, et al. (2009) Relationship between body fat mass and free fatty acid kinetics in men and women. Obesity (Silver Spring) 17, 1872-1877.

26. Wilding JP (2007) The importance of free fatty acids in the development of type 2 diabetes. Diabet Med 24, 934-945.

27. Gibbons GF, Islam K \& Pease RJ (2000) Mobilisation of triacylglycerol stores. Biochim Biophys Acta 1483, 37-57.

28. Avogaro A, Nosadini R, Bier DM, et al. (1990) Ketone body kinetics in vivo using simultaneous administration of acetoacetate and 3-hydroxybutyrate labelled with stable isotopes. Acta Diabetol Lat 27, 41-51. 